\title{
Quantum Zeno Effect Explains Magnetic-Sensitive Radical-Ion-Pair Reactions
}

\author{
I. K. Kominis* \\ Department of Physics, University of Crete, Heraklion 71103, Greece and \\ Institute of Electronic Structure \& Laser, Foundation for Research and Technology, Heraklion 71110, Greece
}

(Dated: October 23, 2018)

\begin{abstract}
Chemical reactions involving radical-ion pairs are ubiquitous in biology, since not only are they at the basis of the photosynthetic reaction chain, but are also assumed to underlie the biochemical magnetic compass used by avian species for navigation. Recent experiments with magnetic-sensitive radical-ion pair reactions provided strong evidence for the radical-ion-pair magnetoreception mechanism, verifying the expected magnetic sensitivities and chemical product yield changes. It is here shown that the theoretical description of radical-ion-pair reactions used since the 70's cannot explain the observed data, because it is based on phenomenological equations masking quantum coherence effects. The fundamental density matrix equation derived here from basic quantum measurement theory considerations naturally incorporates the quantum Zeno effect and readily explains recent experimental observations on low- and high-magnetic-field radical-ion-pair reactions.
\end{abstract}

PACS numbers: $82.20 .-\mathrm{w}$

\section{INTRODUCTION}

Recent experiments [1, 2] on the excitonic energy transfer taking place in photosynthetic reactions have provided a tangible glimpse of quantum coherence effects being at play in biological systems. The possibility of quantum physics underpinning biological systems beyond the structural aspect has been entertained for a long time. It has also been clear [3, 4] that in the decoherence-prone biological environment, some sort of protection of the quantum coherence must be at work before any effects related to the latter can surface. We will here show that a familiar biophysical system, namely radical-ion pairs and their reactions, exactly fulfills the aforementioned requirements and exhibits effects known from quantum physics experiments on well-isolated atomic systems and accounted for by quantum measurement theory.

Radical-ion pairs, created by a charge transfer from a photo-excited donor-acceptor molecular dyad, are central in the reaction chain taking place in the photosynthetic reaction center [5, 6]. The magnetic interactions [7, 8] of the two unpaired electrons in the radical-ionpair with external magnetic fields and internal hyperfine magnetic fields add another layer of complexity in the charge-transfer chain-reactions that convert the absorbed photon energy to chemical energy vital for further biological function. Besides photosynthesis, magneticsensitive recombination reactions of radical-ion pairs are also assumed to underlie the biochemical magnetic compass [9, 10] used by avian and possibly other species for navigation in the geomagnetic field [11, 12, 13, 14]. Furthermore, radical-ion-pairs are also understood to participate in charge transfer reactions in DNA helices [15, 16]. It is thus clear that radical-ion pairs are found in several systems of biological significance, and the complexity of

*Electronic address: ikominis@iesl.forth.gr their dynamics has been attacked from a wide range of theoretical and experimental disciplines.

The time evolution of radical-ion-pair reactions is governed by two distinct processes. The singlet (unpaired electron spins anti-aligned) and triplet (unpaired electron spins aligned) states of the radical-ion-pair and the interconversion between them brought about by the magnetic interactions [7] are one piece of the dynamics. The other is the spin-state dependent charge-recombination of the radical-ion-pair. Singlet pairs recombine only to singlet neutral molecules and triplet pairs recombine only to triplet neutral molecules.

However, since the 1970's, the theoretical treatment (reviewed in [17]) of radical-ion-pair reactions has been based on semi-classical/phenomenological equations involving the density matrix describing the pair's quantum state, not unlike the early rate-equation description of matter-light interactions. The latter is now known to mask important effects related to quantum coherence, which become transparent only in light of the full-blown quantum-mechanical description of atom-photon interactions [18]. It is the recombination process that has so far been treated phenomenologically in a single density matrix equation simultaneously describing the magnetic interactions within the radical-ion-pair. In light of quantum measurement theory applied to radical-ion-pair recombination reactions, it is here shown that this biological system manifests quantum coherence, quantum jumps, the quantum Zeno effect and in principle quantum correlations, that is, the full machinery of quantum information science effects and concepts.

The article is organized as follows. In the following section we will present the shortcomings of the phenomenological master equation describing radical-ion-pair reactions so far while in Section III we put forward the quantum-mechanically consistent master equation. In Section IV we comment on spin relaxation, the presence of which has in many cases conspired with the phenomenological treatment of the dynamics to produce a 


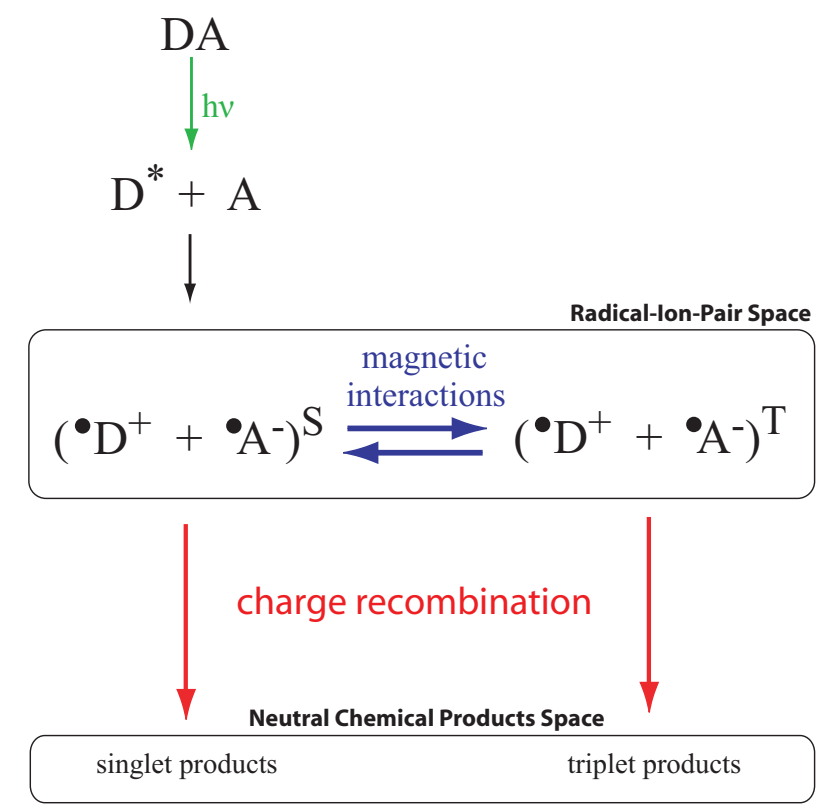

FIG. 1: A simple schematic of radical-ion-pair reaction dynamics. A photon excites a donor-acceptor molecular dyad, which after a charge transfer process creates a radical-ionpair. The magnetic interactions of the two unpaired electrons (two dots) with the external magnetic field and the molecule's magnetic nuclei bring about a coherent singlet-triplet conversion. This would go on forever if it were not for the chargerecombination process that takes radical-ion pairs to neutral products. Angular momentum conservation enforces spin selectivity of the recombination, i.e. singlet (triplet) radical-ion pairs recombine to singlet (triplet) chemical products.

description consistent with experiments. In Section V we use the fundamental quantum dynamic description of radical-ion-pair reactions put forward in Section III to reproduce recent experimental data by Hore and coworkers [19] that clearly manifest quantum coherence effects not accounted for by the phenomenological theory. Finally, based on quantum measurement theory, the derivation of the fundamental master equation describing radical-ion-pair reactions, which is done along the lines presented [20, 21, 22, 23] by Milburn, Wiseman and co-workers on a rather similar system, namely coupled quantum dots, is presented in Section VI.

\section{PHENOMENOLOGICAL MASTER EQUATION}

The phenomenological or semi-classical density matrix equation that has been used so far to describe the dynamics of radical-ion-pair reactions, schematically depicted in Fig. 1, is

$$
\frac{d \rho}{d t}=-i\left[\mathcal{H}_{m a g}, \rho\right]-k_{S}\left(\rho Q_{S}+Q_{S} \rho\right)-k_{T}\left(\rho Q_{T}+Q_{T} \rho\right)
$$

The first term describes the unitary evolution due to the magnetic Hamiltonian $\mathcal{H}_{\text {mag }}$, and the other two terms take into account the population loss due to singlet and triplet state recombination, respectively. The dimension of the density matrix $\rho$ is determined by the number and the nuclear spin of the magnetic nuclei in the radical-ion-pair. It is given by $\operatorname{dim}=4 n$, where 4 is the spin multiplicity of the two unpaired electrons and $n=\left(2 I_{1}+1\right) \times\left(2 I_{2}+1\right) \times \ldots \times\left(2 I_{j}+1\right)$ is the spin multiplicity of the $j$ magnetic nuclei existing in the donor and acceptor molecules. The Hermitian operators $Q_{S}$ and $Q_{T}$ are the singlet and triplet projection operators, respectively, given by

$$
\begin{aligned}
Q_{S} & =1 / 4-\mathbf{s}_{1} \cdot \mathbf{s}_{2} \\
Q_{T} & =3 / 4+\mathbf{s}_{1} \cdot \mathbf{s}_{2}
\end{aligned}
$$

where $\mathbf{s}_{1}$ and $\mathbf{s}_{2}$ are the spins of the two unpaired electrons (obviously $Q_{S}$ and $Q_{T}$ have to be properly written as 4n-dimensional matrices operating in the $4 \mathrm{n}$ dimensional Hilbert space of the particular radical-ionpair in consideration). The singlet and triplet projection operators are orthogonal, $Q_{S} Q_{T}=Q_{T} Q_{S}=0$ and add up to unity, $Q_{S}+Q_{T}=\mathbf{1}$, where $\mathbf{1}$ is the 4 n-dimensional unit matrix. Finally, being projection operators, $Q_{S}$ and $Q_{T}$ satisfy the relations $Q_{S}^{2}=Q_{S}$ and $Q_{T}^{2}=Q_{T}$, respectively.

It is usually considered that the radical-ion-pair starts out in the singlet state, so that we can write for the initial density matrix $\rho(t=0)=Q_{S} / n$, where $n$ is the nuclear spin multiplicity. We let $S(t)=\operatorname{Tr}\left\{\rho(t) Q_{S}\right\}$ and $T(t)=\operatorname{Tr}\left\{\rho(t) Q_{T}\right\}$ denote the time-dependent probability to find the radical-ion-pair in the singlet and triplet state. Due to the structure of (10), the trace of the density matrix decays exponentially to zero, since from (1) it follows that

$$
\frac{d \operatorname{Tr}\{\rho\}}{d t}=-2 k_{S} S-2 k_{T} T
$$

This is the main problem of the phenomenological equation (11), i.e. in order to describe population loss from the radical-ion-pair space into the neutral chemical products space due to charge recombination, the normalization of the density matrix is forced to an exponential decay. All coherences and populations are consequently also forced "by hand" to follow an exponential decay, thus eliminating the actual presence of quantum coherence effects.

What has not been realized so far, however, it that the tunneling taking place in the charge-recombination process is fundamentally a selective quantum measurement continuously interrogating the radical-ion-pair's spin state. If treated as such, the recombination process reveals well-known effects from the realm of quantum measurements, such as the quantum Zeno effect [24, 25], long-lived quantum coherences and quantum jumps [26].

Quantum Zeno effects appear in several physical systems, some of which are very similar to radical-ion-pairs, like the ortho-para conversion in molecular spin isomers 
27], ultra-cold atom tunneling through optical potentials [28], or the suppression of transverse spin-relaxation due to spin-exchange collisions in dense alkali-metal vapors [29, 30]. In the latter case, atomic spin-exchange collisions, of the form $\mathbf{s}_{1} \cdot \mathbf{s}_{2}$, where $\mathbf{s}_{1}$ and $\mathbf{s}_{2}$ are the electron spins of the two colliding atoms, probe the atomic spin state. When the collision rate (measurement rate) exceeds the intrinsic frequency scale of the system, which is the Larmor frequency of spin precession in the applied magnetic field, the effective decay rate of the spin coherence is suppressed, a phenomenon that has led to the development of new ultra-sensitive atomic magnetometers [31, 32]. The radical-ion-pair tunneling into the neutral state is essentially a scattering process 33,34 , not unlike atomic collisions, that performs a measurement of the pair's spin state, since tunneling to singlet (triplet) products can only proceed if the two unpaired electrons are in the singlet (triplet) spin state. Quantum Zeno effects have been extensively analyzed in the literature [35, 36], both with respect to pertaining physical systems, as well as the general conditions leading to the quantum Zeno effect or its inverse, the anti-Zeno effect [37].

\section{QUANTUM MEASUREMENT THEORY DESCRIPTION OF RADICAL-ION-PAIR REACTIONS}

Using standard quantum measurement theory [39] it readily follows (as shown in Section VI in detail) that the fundamental density matrix equation describing radicalion-pair reactions and the associated magnetic-field effects is

$$
\begin{aligned}
\frac{d \rho}{d t}=-i\left[\mathcal{H}_{m a g}, \rho\right] & -k_{S}\left(\rho Q_{S}+Q_{S} \rho-2 Q_{S} \rho Q_{S}\right) \\
& -k_{T}\left(\rho Q_{T}+Q_{T} \rho-2 Q_{T} \rho Q_{T}\right)
\end{aligned}
$$

which is the same as the (1), apart from the terms $2 Q_{S} \rho Q_{S}$ and $2 Q_{T} \rho Q_{T}$. It is these terms that are responsible for the quantum effects that become important in the parameter regime where the recombination rates are larger than the magnetic interactions frequency scale. Since $Q_{S}+Q_{T}=1$, (5) can be simplified to

$$
\frac{d \rho}{d t}=-i\left[\mathcal{H}_{m a g}, \rho\right]-k\left(\rho Q_{S}+Q_{S} \rho-2 Q_{S} \rho Q_{S}\right)
$$

where $k=k_{S}+k_{T}$. The physical interpretation of (6) is that both singlet and triplet recombination channels essentially "measure" the same observable, $Q_{S}$, with a total measurement rate $k_{S}+k_{T}$. In other words, the singlet (triplet) recombination channel is interrogating the radical-ion-pair whether it is in the singlet (triplet) state. But being in the triplet is not being in the singlet $\left(Q_{S}+Q_{T}=1\right)$, thus both channels measure the same observable. This physical interpretation of the actual dynamics is completely lacking from the phenomenological equation (1). The density matrix equation (6) has the property that the normalization of the density matrix does not change, i.e. $\operatorname{Tr}\{\rho\}=S(t)+T(t)=1$ at all times. It is here noted that as early as 1976, Haberkorn [38] arrived at (6) based on semi-quantitative arguments, but did not further consider it, exactly because it does not seem to describe population loss from the radicalion-pair space into the reaction products space due to recombination, since $\operatorname{Tr}\{\rho\}=1$ at all times. All works henceforth have used the phenomenological equation (1).

To take recombination into account, we need another stochastic equation involving quantum jumps [39] out of the radical-ion-pair space into the neutral chemical products space. These are given by the probability of the singlet and triplet channel recombination,

$$
\begin{aligned}
& p_{S}=2 k_{S}\left\langle Q_{S}\right\rangle d t \\
& p_{T}=2 k_{T}\left\langle Q_{T}\right\rangle d t
\end{aligned}
$$

The physical interpretation of the above equations is the following: $p_{S}\left(p_{T}\right)$ is the probability that the radical-ionpair recombines into the singlet (triplet) channel in the time interval between $t$ and $t+d t$. Both $p_{S}$ and $p_{T}$ are obviously positive-definite numbers (the expectation values of $Q_{S}$ and $Q_{T}$ range between 0 and 1). In other words, Haberkorn obtained the correct density matrix evolution equation, missing, however, the quantum jump equations (7) and their physical interpretation, as the conceptual underpinnings of quantum measurement theory and open quantum systems were lacking at the time. In Section $\mathrm{V}$ on the explanation of recent experimental data we will explicitly explain how the quantum dynamic evolution described by (6) and (77) is actually simulated.

Thus the fundamental quantum dynamical evolution of radical-ion-pair recombination reactions is given by (6) together with (7). To summarize the fundamental difference of the quantum description based on (6) and (7) versus the phenomenological (1) we note that (1) is such that it continuously removes population from the singlettriplet subspace into the chemical product space, and does so independently for each recombination channel. In reality however, the actual evolution of the radicalion-pair's spin state is given by (6) (both channels affect the spin state evolution) and the molecules recombine in random times and in jump-like fashion according to (7). Thus even if the radical-ion-pair starts out in the singlet state, a high triplet recombination rate will significantly affect the spin state of the molecule, well before it has time to acquire a triplet character through the magnetic interactions. This physical statement is not embodied in the phenomenological equation (11).

\section{SPIN RELAXATION}

There are two points that need to be commented on before proceeding to present numerical simulations and the explanation of recent experimental data. The creation 

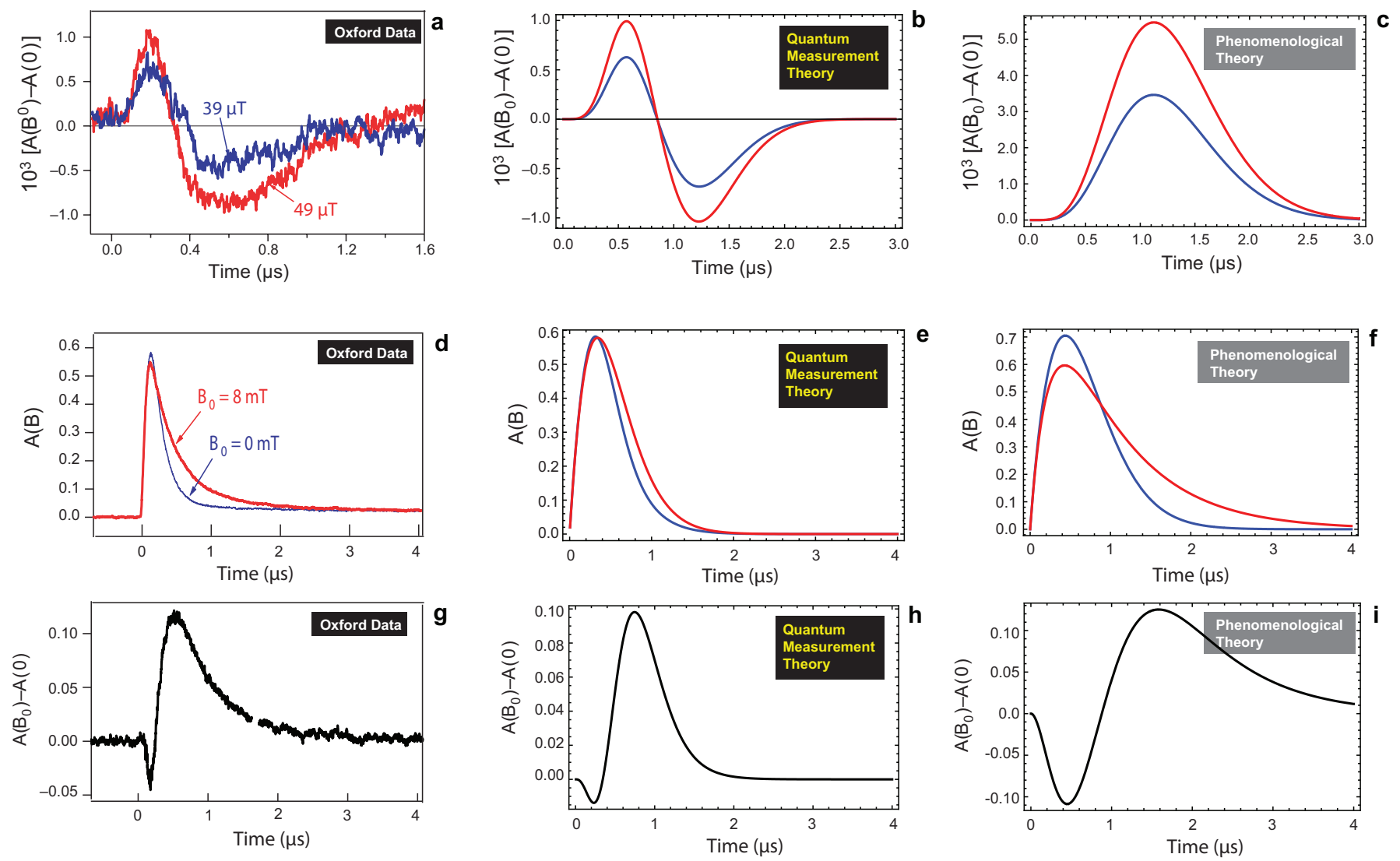

FIG. 2: Magnetic Field Effect, Experimental Data and Theoretical Calculation. (a) Measured [19] magnetic field effect for two different magnetic fields $B=49 \mu \mathrm{T}$ (red curves) and $B=39 \mu \mathrm{T}$ (blue curves). (b) Reproduction of data from quantum measurement theory based on (6) and (7) with a simple one-nuclear-spin (spin-1/2) radical-ion-pair model with a diagonal hyperfine tensor given by $a_{x}=8 \mu \mathrm{s}^{-1}, a_{y}=2 \mu \mathrm{s}^{-1}$ and $a_{z}=0$. The recombination rates used are $k_{S}=0.05 \mu \mathrm{s}^{-1}$ and $k_{T}=3.5 \mathrm{\mu s}^{-1}$. (c) Reproduction of data from the phenomenological theory (1) with the same parameters as in (b). (d) Measured [19] transient absorption traces at zero and high magnetic field and (g) corresponding magnetic field effect. (e) and (h) Reproduction of data from quantum measurement theory based on (6) and (7) with the same parameters as in (b). (f) and (i) Reproduction of data from the phenomenological theory (11) with the same parameters as in (b). For the reproduction of the high field data we had to consider the creation dynamics of radical-ion pairs (mentioned in Section IV) with $k_{c r}=4 \mu \mathrm{s}^{-1}$ and a high field relaxation rate $k_{B, s r}=1.0 \mu \mathrm{s}^{-1}$. As explained in Section IV, due to the spin relaxation present at high fields, the qualitative disagreement between data and phenomenological theory is not that pronounced as at low fields.

of radical-ion pairs does not happen instantaneously, but at a given rate $k_{c r}$. This can be modeled by adding a source term to the density matrix equations, $k_{c r} e^{-k_{c r} r}$, the time-integral of which is equal to 1 . This is not a fundamental point and will henceforth be neglected, i.e. we will assume that the rate $k_{c r}$ is much larger than all other rates of the problem, unless noted otherwise. Second and more important is the effect of spin-relaxation. This can be modeled by a term $-k_{s r}\left(\rho-\rho_{0}\right)$, where $k_{s r}$ is the relaxation rate and $\rho_{0}=1 / 4 n$ is the fully mixed density matrix, i.e. a diagonal $4 n$-dimensional matrix with diagonal elements equal to $1 / 4 n$. In the phenomenological equation (11) the analogous spin-relaxation term should read $-k_{s r} \rho$, since by design equation (10) has $\rho=0$ as the infinite-time solution, hence the relaxation term should work towards the same steady state. We will "turn on" spin relaxation by setting $k_{s r} \neq 0$, to show that in the presence of spin relaxation the results following from the quantum-mechanical description based on (6) and (7) naturally blend in with the ones derived from (11). This is the main reason why so far the phenomenological density matrix equation (10) has provided a more or less consistent theoretical description of experimental observations. Obviously, the charge recombination process itself induces spin relaxation, and this is what is described by (6). By the rate $k_{s r}$ we describe all other spin relaxation effects, for example spin-lattice relaxation. It is also noted that diffusion or other effects add another layer of complexity to the dynamics described by (6) and (7) and will not be considered here. In other words, equations (6) and (7) are the fundamental dynamical equations describing magnetic-sensitive radical-ion-pair reactions in the idealized case of negligible diffusion. This is not unrealistic, since at low temperatures (as for example in the experiment reported in Ref. [19]) the effects of diffusion and more importantly, spin relaxation, are suppressed. 


\section{EXPLANATION OF EXPERIMENTAL DATA}

We will now show that the quantum-mechanical analysis of the radical-ion-pair reactions put forward in Section III seamlessly explains recent experimental data, while the phenomenological theory is unable to do so. In the experiment recently reported [19] by Hore and co-workers, a carotenoid-porphyrin-fullerene (CPF) triad was used to study the magnetic field effect on the radicalpair's recombination dynamics. We have performed numerical simulations using the simplest physically realizable radical-ion-pair model, that containing just one spin$1 / 2$ magnetic nucleus. In this case the density matrix is 8-dimensional and the magnetic Hamiltonian reads

$$
\mathcal{H}_{\text {mag }}=\omega\left(s_{1 z}+s_{2 z}\right)+\mathbf{I} \cdot \mathbf{A} \cdot \mathbf{s}_{1}
$$

where we considered the external magnetic field to be along the $z$-axis, $\mathbf{B}=B \hat{\mathbf{z}}$, and the electron Larmor frequency parameter (the real Larmor frequency is two times higher) is $\omega=\gamma_{e} B$, with $\gamma=1.4 \mathrm{MHz} / \mathrm{G}$. The hyperfine coupling tensor $\mathbf{A}$ couples the single nuclear spin existing in either the donor or the acceptor molecule with the corresponding unpaired electron. Anisotropic hyperfine interactions embodied in the hyperfine tensor are responsible for the directional sensitivity of the reaction, i.e. the sensitivity of the reaction yields on the angle between the external magnetic field and the local coordinate frame defined by the hyperfine tensor. In the experiment reported in [19] the angular dependence of the reaction has also been measured, and can also be reproduced theoretically from the quantum dynamic description of the reaction, however, we will present this topic in a different manuscript.

By direct numerical integration of either (11) or (6) and (7) we calculate the population of the triad molecules in the singlet-triplet subspace at time $t$. This population is what is measured in the transient absorption measurement. The "magnetic-field effect" is the difference $(S(t)+T(t))_{B}-(S(t)+T(t))_{B=0}$, i.e. the difference of the measured transient absorption at a magnetic field $B$ from that at zero field. For the sake of completeness we note that while the phenomenological predictions are obtained by a direct integration of (11), the quantum dynamic predictions based on (6) and the quantum jump equation (7) are obtained as follows: we start with $N_{0}$ radical-ion pairs, and in each time step the number of existing radical-ion-pairs $N(t)$ evolves according to

$$
N(t)=N(t-d t)\left(1-p_{S}-p_{T}\right)
$$

i.e. the number of radical-ion-pairs disappearing to the chemical product space (either singlet or triplet) between time $t-d t$ and time $t$ is $N(t-d t)$ times the probability to jump to a recombined state (either singlet or triplet) during the time interval $d t$. This probability is just $p_{S}+p_{T}$, where $p_{S}$ and $p_{T}$ are given by (7). Along the reaction, the density matrix $\rho$ describing the spin state of the radicalion-pair is evolved according to (6).
We take the radical-ion-pair to be in the singlet state initially, i.e. $\rho(t=0)=Q_{S} / 2$ (in general for a singlet initial state it is $\rho(t=0)=Q_{S} / n$, where $n$ is the nuclear spin multiplicity, which in this case of one spin$1 / 2$ nucleus is $n=2$ ). In Fig. 2a we show the measured magnetic field effect at low magnetic fields (earth's field), while in Fig. 2 $\mathrm{b}$ we fully reproduce the data using the quantum dynamics description of (6) and (7), whereas Fig 2k shows that the phenomenological equation (11) is not capable of reproducing the observed magnetic field effect with the same parameters, and the same is true for any choice of the system's parameters.

It is seen that the quantum dynamic description based on (6) and (7) agrees with the data both qualitatively and quantitatively (the magnitude of the effect), whereas the opposite is true for the phenomenological predictions shown in Fig. 2k. It is noted that the time-scale of the measured magnetic field effect is missed by about a factor of 2 , since in reality the molecule's dynamics are determined by tens of nuclear spins, whereas here we have considered just one nuclear spin. It is also important to note the following: in Fig. 20 we reproduce the data using a small value for the singlet-channel recombination rate and a much larger value for the triplet-channel recombination rate, while the molecule starts out in the singlet state. This is exactly the parameter regime where the quantum Zeno effect is actually manifested, i.e. as mentioned before, the high triplet recombination rate has a tangible effect on the spin dynamics, as it appears in the total measurement rate $k=k_{S}+k_{T}$. This is a regime that the phenomenological theory fails to account for even at the qualitative level.

We will now demonstrate that if we take into account a possible presence of spin-relaxation, the magnetic field effect is suppressed both in the phenomenological description and in the quantum-mechanical, and both descriptions qualitatively merge into each other. The presence of spin-relaxation effects is one reason why the phenomenological reaction dynamics of equation (1) have been relatively successful until now. This is expected since in general relaxation effects tend to equalize level populations and damp quantum coherences, adversely affecting the precision of any spectroscopic measurement, such as the determination of the applied magnetic field. We will further show that the bi-phasic response observed in the data (Fig. 2a) and reproduced theoretically (Fig. 2b) fades away when we "turn on" spin relaxation, and resembles the response produced by the phenomenological equation (11). The reason that the experiment by Maeda et al [19] manifests effects not accountable by the density matrix equation used until now is the suppressed presence of spin-relaxation effects at the low temperatures at which the experiment was carried out. Indeed, in Fig. 3 we turn on spin-relaxation and it is evident that the magnetic field effect derived from (6) and (7) resembles that derived from (1) as the spin relaxation rate $k_{s r}$ is 

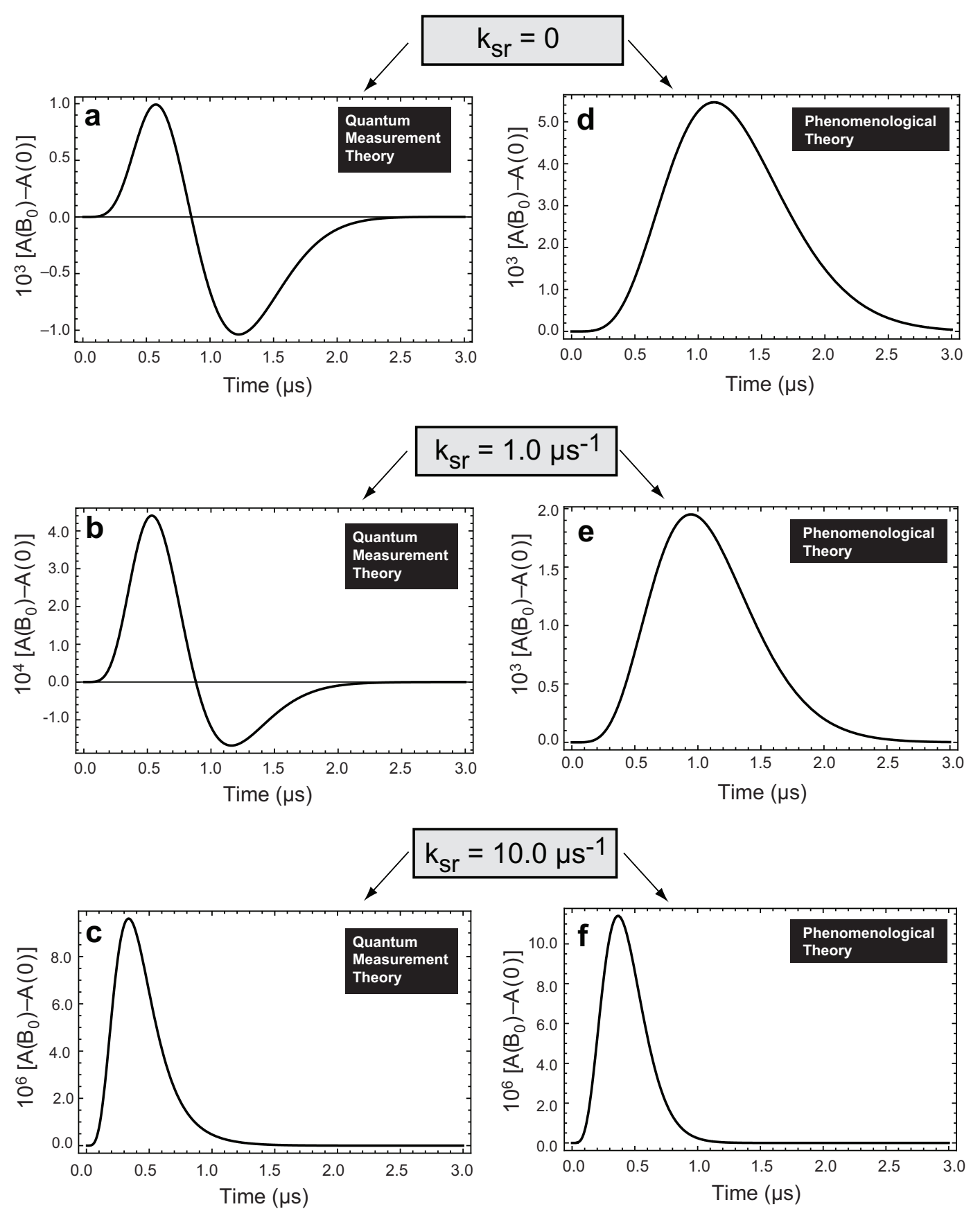

FIG. 3: Magnetic Field Effect and Spin Relaxation. The magnetic field effect is simulated at a magnetic field $B=49 \mu$ T. Traces a,b,c have been produced with the quantum dynamics given by (6) and (7), while traces d,e,f have been produced by the phenomenological equation (11). The same calculations have been done for three different values of the relaxation rate $k_{s r}=0,1,10 \mu \mathrm{s}^{-1}$. Plot (a) is the same as plot (b) of Fig. 2, i.e. the one reproducing the low-field data of [19]. It is seen that by increasing the spin relaxation rate, the two theories produce qualitatively and quantitatively similar results, i.e. for increasing $k_{s r}$ the bi-phasic response of the magnetic-field-effect is suppressed and the magnitude of the effect is roughly the same in both theories. It is thus clear that the presence of spin relaxation has in many cases masked the insufficiency of the phenomenological description and made it appear consistent with experiments. The other way around, it is clear that the low temperatures used in the recent experiment by Hore and co-workers [19] has suppressed spin-relaxation effects to the extent that the true quantum nature of radical-ion-pair reactions could surface. 
increased.

Finally, also the high-field data of [19] can be explained with the same magnetic Hamiltonian used to reproduce the low-field data shown in Fig. 2a. To this end we take into account the creation of radical-ion pairs, which does not take place instantaneously, but at a given rate $k_{c r}$, modelled by the term $d N / d t=N_{0} k_{c r} e^{-k_{c r} t}$, where $N_{0}$ is the total number of molecules. In Fig. $2 \mathrm{~d}$ we show the experimental results for the transient absorption at a magnetic field of $B=0$ and $B=8 \mathrm{mT}$, with the quantum measurement calculation shown in Fig. 2 e and the phenomenological prediction in Fig. 2f. Similarly, Figures $2 \mathrm{~b}, \mathrm{~h}, \mathrm{i}$ show the data and theoretical predictions for the magnetic field effect, i.e. the difference of the traces shown in Figures 2 $2 \mathrm{~d}$,e and $\mathrm{f}$. The initial negative response is due to non-zero spin-relaxation at high magnetic fields, which we model as described previously and which has been mentioned to exist in [19]. It is interesting to note the fact that the data reproduction by quantum measurement theory is capable of picking up this small spin relaxation $\left(k_{s r}=1 \mu \mathrm{s}^{-1}\right)$ existing at high magnetic fields. Taking into account the simplicity of the magnetic Hamiltonian used for the calculations, the agreement between experimental data and the predictions of quantum measurement theory is excellent, as both the absolute value of the transient absorption as well as the strength of the magnetic field effect and the time scale of the phenomenon are perfectly reproduced. It is also interesting to note that at high magnetic fields the disagreement between data and the predictions of the phenomenological theory is not that pronounced as at low fields. This is exactly due to the small spin relaxation present at high fields.

\section{DERIVATION OF MASTER EQUATION}

In Figure 4 we depict in more detail the generic model for the radical-ion-pair creation and recombination dynamics shown in Fig. 1. A donor-acceptor molecule DA is photo-excited $\left(\mathrm{D}^{*} \mathrm{~A}\right)$ and a subsequent charge-transfer creates the radical-ion-pair $\left(\mathrm{D}^{+} \mathrm{A}^{-}\right)$. The singlet and triplet states of the radical-ion-pair $\left({ }^{S} \mathrm{D}^{+} \mathrm{A}^{-},{ }^{T} \mathrm{D}^{+} \mathrm{A}^{-}\right)$ are split by internal magnetic interactions of the radicalion-pair's two unpaired electrons with external magnetic fields and internal hyperfine couplings. The radical-ionpair is initially created in the singlet state, which is not an eigenstate of the magnetic Hamiltonian, and therefore a singlet-triplet (S-T) coherent mixing commences. A tunneling event into a vibrationally-excited state of the neutral recombined molecule DA, which quickly decays into the ground state, completes the photon-initiated radicalion-pair reaction.

As is well known [40, 41], electron transfer in radicalion-pair recombination reactions is fundamentally a quantum-mechanical tunneling process. We will show that this process constitutes a continuous quantum measurement of the pair's spin state. Like every quantum

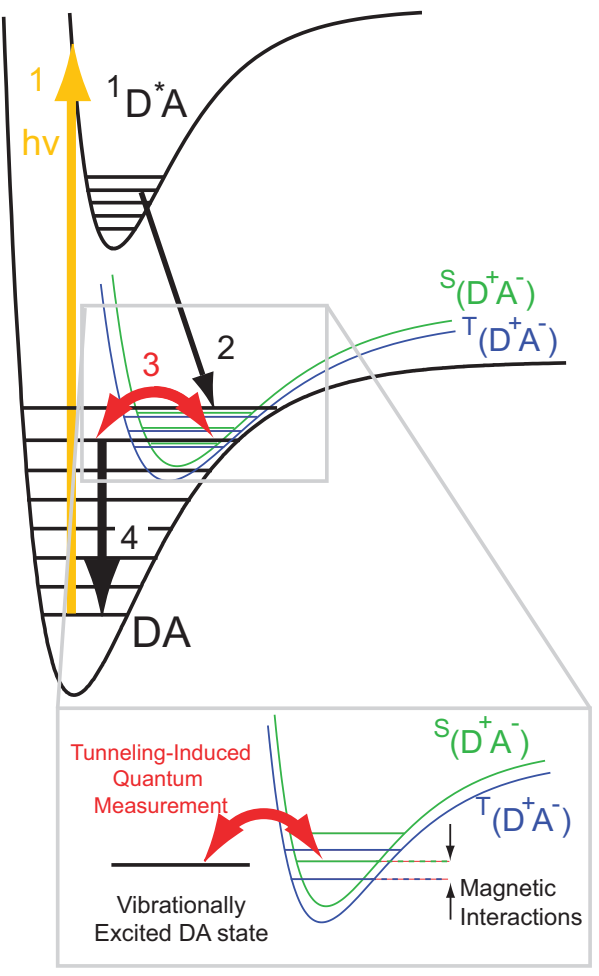

FIG. 4: Generic level structure and recombination dynamics in a radical-ion-pair, taking place in four steps: 1, photoexcitation, 2, radical-ion-pair creation, 3, tunneling-induced quantum measurement of the pair's spin state and 4 , final decay to the ground state. Only the singlet reservoir of vibrationally excited states (of the neutral molecule DA) is shown for simplicity. Another such reservoir exists for the triplet chemical products.

measurement, this one is no exception to the rule that measurements performed on a quantum system lead to decoherence [42]. However, under appropriate conditions involving the measurement rate and the intrinsic (magnetic) frequency scale of the radical-ion-pair, the quantum Zeno effect [24, 43] appears and leads to physical consequences completely against the intuition that has developed over the years based on the phenomenological description of (11).

In the following, we are going to capitalize on the remarkably strong analogy between radical-ion-pairs and yet another physical system, namely two coupled quantum dots [20, 21, 22, 23, 44] being continuously interrogated by a point contact. This analogy is schematically depicted in Fig. 5. We are going to identify the analogous physical observables of the two systems and then derive the corresponding evolution equation describing radical-ion-pair reactions. The electron hopping between the two dots is the analog of the S-T coherent mixing taking place in the radical-ion-pair, whereas the measurement performed by the point contact corresponds to the spin-state-dependent electron tunneling into an adjacent excited state of the neutral molecule. Radical-ion pairs in the singlet (triplet) state will tunnel to a nearby 
a
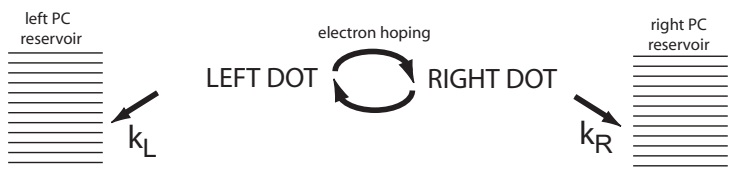

b

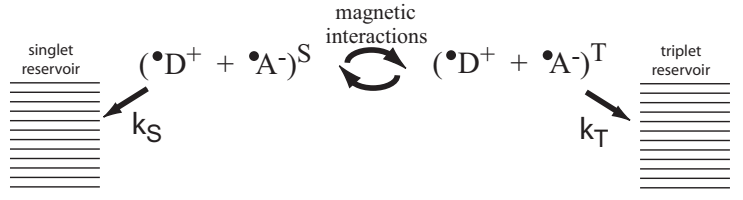

FIG. 5: Analogy between coupled quantum dots and radicalion-pair reactions. (a) Schematic of coupled quantum dots interrogated by a double point contact (PC). (b) Schematic of radical-ion-pair with the singlet and triplet recombination reservoirs.

empty reservoir of singlet (triplet) vibrationally excited states, the annihilation and creation operators of which are denoted by $a(b)$ and $a^{\dagger}\left(b^{\dagger}\right)$, respectively. The corresponding Hamiltonian is

$$
\mathcal{H}_{\text {res }}=\sum_{a} \omega_{a} a^{\dagger} a+\sum_{b} \omega_{b} b^{\dagger} b
$$

As explained before, the radical-ion-pair consists of two unpaired electrons, one at the site of the donor (D) and one at the site of the acceptor (A). We denote by $c$ and $c^{\dagger}$ the fermion annihilation and creation operators of the electron at the acceptor site. Obviously, if $\left\langle c^{\dagger} c\right\rangle=1$ we have a radical-ion-pair, if however, $\left\langle c^{\dagger} c\right\rangle=0$, we have a neutral recombined molecule. The Hamiltonian of the radical-ion-pair thus reads

$$
\mathcal{H}_{\text {rip }}=c^{\dagger} c\left(\epsilon+\mathcal{H}_{\text {mag }}\right)
$$

where $\mathcal{H}_{\text {mag }}$ is the magnetic Hamiltonian operating in the spin space of the radical-ion-pair (for a single nuclear spin it is given in (8) and it is readily generalized for any number of nuclear spins by adding more hyperfine interaction terms) and $\epsilon$ the energy of the radical-ionpair with respect to the ground state neutral molecule. Lastly, the tunneling Hamiltonian reads

$$
\mathcal{H}_{T}=\sum_{a} T_{a} c a^{\dagger} Q_{S}+\sum_{b} T_{b} c b^{\dagger} Q_{T}+\text { c.c. }
$$

The interpretation of the first term is that if the radicalion-pair is in the spin singlet state it can charge recombine, reducing the radical-ion-pair occupation number to zero and creating an occupied vibrationally excited state of the singlet reservoir. Similarly for the triplet state in the second term. In summary, the total Hilbert space consists of the singlet and triplet reservoirs, the occupation of the radical-ion-pair's acceptor electron and the spin space of the radical-ion-pair. For example, in the case of singlet-channel recombination, the initial and final quantum states coupled by $\mathcal{H}_{T}$ would look like
$\left|\Psi_{i}\right\rangle=|\{0\}, 1\rangle|\chi\rangle$ and $\left|\Psi_{f}\right\rangle=|\{n\}, 0\rangle\left|\chi_{s}\right\rangle$, where the first set of quantum numbers $\{n\}$ is the excitation of the vibrational reservoir, the second is the electron occupation number of the acceptor site, and $|\chi\rangle$ is the spin state of the radical-ion-pair (two electrons plus nuclei), while $\left|\chi_{s}\right\rangle$ is the electron spin singlet state of the neutral donor electrons with the nuclear spin state being the same in $|\chi\rangle$ and $\left|\chi_{s}\right\rangle$. The tunneling amplitudes are [40] $T_{i}=V_{i} f_{i}$ $(i=a, b)$, where $V_{i}$ is the electronic matrix element and $f_{i}$ the vibrational overlap between the nuclear wavefunctions of the reservoir states $|i\rangle$ and the radical-ion-pair.

A very similar set of Hamiltonians has already been treated by Milburn, Wiseman and co-workers [20, 21, 22. for the case of quantum dots, and we will here proceed along the same lines to derive the master equation for radical-ion pairs. We write the total Hamiltonian as $\mathcal{H}=$ $\mathcal{H}_{0}+\mathcal{V}$, where

$$
\mathcal{H}_{0}=c^{\dagger} c \epsilon+\mathcal{H}_{\text {res }}
$$

and the perturbation $\mathcal{V}$ is

$$
\mathcal{V}=c^{\dagger} c \mathcal{H}_{m a g}+\mathcal{H}_{T}
$$

and move to the interaction picture defined by $\mathcal{H}_{0}$, in which the perturbation is $\mathcal{V}_{I}=e^{i \mathcal{H}_{0} t} V e^{-i \mathcal{H}_{0} t}$ and it follows that

$$
\begin{aligned}
\mathcal{V}_{I}(t) & =c^{\dagger} c \mathcal{H}_{\text {mag }} \\
& +\sum_{a} T_{a} c a^{\dagger} Q_{S} e^{i\left(\omega_{a}-\epsilon\right) t}+\text { c.c. } \\
& +\sum_{b} T_{b} c b^{\dagger} Q_{T} e^{i\left(\omega_{b}-\epsilon\right) t}+\text { c.c. }
\end{aligned}
$$

The proof of (15) is given in the Appendix. We will now apply time-dependent perturbation theory up to $2^{\text {nd }}$ order. The density matrix of the total system (spin state of radical-ion-pair + electron + reservoir states) is denoted by $W$. The density matrix of interest describing the radical-ion-pair spin state will be $\rho$, and will be calculated by tracing out the other degrees of freedom from $W$. During the time of coherent singlet-triplet mixing, i.e. as long as the radical-ion-pair has not recombined, the reservoir states are empty, which means that the density matrix of the singlet and triplet reservoirs is $\rho_{\text {res }}=\sum_{a}|0\rangle_{a a}\left\langle 0\left|\otimes \sum_{b}\right| 0\right\rangle_{b b}\langle 0|$. For the same reason, the density matrix for the occupation state of the acceptor electron is $\rho_{c}=|1\rangle_{c c}\langle 1|$ and the total density matrix is then given by $W(t)=\rho(t) \otimes \rho_{\text {res }} \otimes \rho_{c}$. Obviously $\rho(t)=\operatorname{Tr}_{\mathrm{a}, \mathrm{b}, \mathrm{c}}\{\mathrm{W}(\mathrm{t})\}$.

Since the tunneling part of the perturbation $\mathcal{V}_{I}$ is linear in the reservoir operators $a, a^{\dagger}, b$ and $b^{\dagger}$, it easily follows that in the $1^{\text {st }}$-order of the perturbation expansion $(\hbar=$ 1)

$$
\begin{aligned}
W(t+d t) & =W(t) \\
& -i d t\left[\mathcal{V}_{I}(t), W(t)\right] \\
& -d t \int_{0}^{t} d t_{1}\left[\mathcal{V}_{I}(t),\left[\mathcal{V}_{I}\left(t_{1}\right), W(t)\right]\right]
\end{aligned}
$$


the terms in $V_{I}$ containing the tunneling amplitudes $T_{a}$ and $T_{b}$ do not contribute, because e.g. $\operatorname{Tr}_{a, b}\left\{\rho_{\text {res }} a\right\}=0$. Since $\operatorname{Tr}_{\mathrm{c}}\left\{\mathrm{c}^{\dagger} \mathrm{c} \mathcal{H}_{\text {mag }} \rho_{\mathrm{c}}\right\}=\left\langle\mathrm{c}^{\dagger} \mathrm{c}\right\rangle \mathcal{H}_{\text {mag }}=\mathcal{H}_{\text {mag }}$, it follows that the $1^{\text {st }}$-order term in (16) results in

$$
\left(\frac{d \rho}{d t}\right)_{1^{\text {st }- \text { order }}}=-i\left[\mathcal{H}_{m a g}, \rho\right]
$$

We will now proceed to calculate the integral appearing in the $2^{\text {nd }}$-order term of (16). In this term we will neglect $\mathcal{H}_{\text {mag }}$ with respect to $\mathcal{H}_{T}$, under the assumption that the measurement dynamics embodied in the tunneling Hamiltonian (12) dominates the magnetic interactions, as is actually the case in the regime where the quantum Zeno effect is manifested. This approximation is validated by the fact that the resulting master equation has the ability to reproduce experimental data. We will show the calculation just for the $a$-reservoir states (the singlet reservoir) in (15), as the term involving the triplet reservoir ( $b$-states) is dealt with in exactly the same way. In the integral appearing in (16), containing $V_{I}$ to $2^{\text {nd }}$-order, terms involving $T_{a}$ from $V_{I}(t)$ and $T_{a}$ from $V_{I}\left(t_{1}\right)$ contain the operator $c^{2}$, which is zero. The only terms that survive are the ones containing $T_{a}$ from the $V_{I}(\mathrm{t})$-term and $T_{a}^{*}$ from the complex conjugate part of the $V_{I}\left(t_{1}\right)$-term. These terms lead (after taking into account the fact that cross-terms involving different reservoir states $a$ and $a^{\prime}$ as well as cross terms involving both reservoirs do not contribute) to an expression of the form

$$
\begin{aligned}
& -d t \sum_{a}\left|T_{a}\right|^{2} \int_{0}^{t} d t_{1} e^{i\left(\omega_{a}-\epsilon\right)\left(t-t_{1}\right)}\left[c a^{\dagger} Q_{S},\left[c^{\dagger} a Q_{S}, W\right]\right] \\
& + \text { c.c. }
\end{aligned}
$$

Taking into account the fact that for the empty reservoir states it holds $\left\langle a^{\dagger} a\right\rangle=0$ and $\left\langle a a^{\dagger}\right\rangle=1$, while for the acceptor electron it is $\left\langle c^{\dagger} c\right\rangle=1$ and $\left\langle c c^{\dagger}\right\rangle=0$, it follows that when tracing out the $a$ and $c$ degrees of freedom from the double commutator in (18), we are left with the operator $\rho Q_{S}-Q_{S} \rho Q_{S}$. The complex conjugate (c.c.) term will then offer $Q_{S} \rho-Q_{S} \rho Q_{S}$, thus in total we obtain the Lindblad form $\rho Q_{S}+Q_{S} \rho-2 Q_{S} \rho Q_{S}$. We finally consider the resonance condition $\omega_{a} \approx \epsilon$ and make the usual approximation $\int_{0}^{t} d t_{1} e^{i\left(\omega_{a}-\epsilon\right)\left(t-t_{1}\right)} \approx 2 \pi \delta\left(\omega_{a}-\epsilon\right)$, leading to the desired result, which after doing the same calculation for the triplet reservoir states $b$, reads

$$
\frac{d \rho}{d t}=-i\left[\mathcal{H}_{m a g}, \rho\right]-k_{S} \mathcal{D}\left[Q_{S}\right] \rho-k_{T} \mathcal{D}\left[Q_{T}\right] \rho
$$

where the super-operator $\mathcal{D}[B]$ acts on the density matrix $\rho$ according to

$$
\mathcal{D}[B] \rho=B^{\dagger} B \rho+\rho B^{\dagger} B-2 B \rho B^{\dagger}
$$

We have thus derived (6), with the recombination rates given by (we have re-introduced $\hbar$ for the consistency of units)

$$
\begin{aligned}
& k_{S}=\left(2 \pi / \hbar^{2}\right) \sum_{a}\left|T_{a}\right|^{2} \delta\left(\epsilon-\omega_{a}\right) \\
& k_{T}=\left(2 \pi / \hbar^{2}\right) \sum_{b}\left|T_{b}\right|^{2} \delta\left(\epsilon-\omega_{b}\right)
\end{aligned}
$$

The resulting master equation (6) is indeed of the Lindblad form that follows from standard quantum measurement theory [39, 42, 45], when the measured observable is $Q_{S}$ and the measurement rate is $k=k_{S}+k_{T}$, leading to the physical interpretation that the spin-dependent recombination process is a continuous quantum measurement of the radical ion pair's spin state.

\section{CONCLUSIONS}

In summary, we have shown that there exists a biological system, radical-ion pairs and their reactions, in which the full machinery of quantum measurement theory can be fruitfully applied, leading to an understanding of quantum effects visible in the laboratory but masked by the phenomenological description of radical-ion-pair reactions employed until now. A fundamental quantum phenomenon, the quantum Zeno effect, familiar from carefully prepared atomic physics experiments, is seen to participate in the complex realm of biologically significant chemical reactions, further opening up the way to the emerging field of quantum biology, i.e. the exploration of the manifestations of quantum phenomena and the application of quantum information concepts in biological systems.

\section{Acknowledgments}

I would like to acknowledge Prof. D. Anglos for several useful and stimulating discussions.

\section{APPENDIX}

\section{Proof of equation (15)}

We will show that $e^{i \epsilon c^{\dagger} c t} c e^{-i \epsilon c^{\dagger} c t}=c e^{-i \epsilon t}$. Since $c$ is a fermion annihilation operator, it is $c^{2}=0$, thus $e^{i \epsilon c^{\dagger} c t} c=$ c. Furthermore, since $\left(c^{\dagger} c\right)^{n}=c^{\dagger} c$ for any integer power $n$, it follows that $e^{-i \epsilon c^{\dagger} c t}=e^{-i \epsilon t} c^{\dagger} c+c c^{\dagger}$, leading to the desired result. In a similar fashion it can be shown that $e^{i \mathcal{H}_{\text {res }} t} a^{\dagger} e^{-i \mathcal{H}_{\text {res }} t}=a^{\dagger} e^{i \omega_{a} t}$ and similarly for the $b$ degrees of freedom, thus follows equation (15). 
[1] G. S. Engel et al., Nature 446, 782 (2007).

[2] H. Lee, Y. C. Cheng and G. R. Fleming, Science 316, 1462 (2007).

[3] P. C. W. Davies, Biosystems 78, 69 (2004).

[4] D. Abbott et al., Fluctuations and Noise Letters 8, C5 (2008).

[5] S. G. Boxer, Biochim. Biophys. Acta 726, 265 (1983).

[6] S. G. Boxer, C. E. D. Chidsey and M. G. Roelofs, Ann. Rev. Phys. Chem. 34, 389 (1983).

[7] C. R. Timmel, U. Till, B. Brocklehurst, K. A. McLaughlan and P. J. Hore, Molec. Phys. 95, 71 (1998);C. R. Timmel, F. Cintolesi, B. Brocklehurst and P. J. Hore, Chem. Phys. Lett. 334, 387 (2001).

[8] C. R. Timmel and K. B. Henbest, Phil. Trans. R. Soc. Lond. A 362, 2573 (2004).

[9] K. Schulten, C. E. Swenberg and A. Weller, Z. Phys. Chem. 111, 1 (1978).

[10] K. Schulten, Adv. Solid State Phys. 22, 61 (1982).

[11] T. Ritz, S. Adem and K. Schulten, Biophys. J. 78, 707 (2000).

[12] W. Wiltschko and R. Wiltscko, J. Comp. Physiol. A 191, 675 (2005).

[13] T. Ritz, P. Thalau, J. B. Phillips, R. Wiltschko R and W. Wiltschko, Nature 429, 177 (2004).

[14] S. Johnsen and K. J. Lohmann, Physics Today 61, 29 (2008).

[15] F. D. Lewis et al., Science 277, 673 (1997).

[16] M. Bixon et al., Proc. Natl. Acad. Sci. USA 96, 11713 (1999).

[17] U. E. Steiner and T. Ulrich, Chem. Rev. 89, 51 (1989).

[18] C. Cohen-Tannoudji, J. Dupont-Roc and G. Grynberg, Atom-photon interactions:basic processes and applications, John Wiley \& Sons, 1998.

[19] K. Maeda et al., Nature 453, 387 (2008).

[20] H. B. Sun and G. J. Milburn, Phys. Rev. B 59, 10748 (1999).

[21] H. M. Wiseman et al., Phys. Rev. B 63, 235308 (2001).

[22] H. S. Goan, G. J. Milburn, H. M. Wiseman and H. B. Sun, Phys. Rev. B 63, 125326 (2001).
[23] N. P. Oxtoby, A Quantum Trajectory Approach to Realistic Measurement of Solid-State Quantum Systems, Ph.D. Thesis, Griffith University, 2006.

[24] B. Misra and E. C. G. Sudarshan, J. Math. Phys. 18, 756 (1977).

[25] P. Facchi and S. Pascazio, Fortschr. Phys. 49, (2001).

[26] M. B. Plenio and P. L. Knight, Rev. Mod. Phys. 70, 101 (1998).

[27] B. Nagels, L. J. F. Hermans and P. L. Chapovsky, Phys. Rev. Lett. 79, 3097 (1997).

[28] S. R. Wilkinson et al., Nature 387, 575 (1997).

[29] W. Happer and H. Tang, Phys. Rev. Lett. 31, 273 (1973).

[30] I. K. Kominis, Phys. Lett. A 372, 4877 (2008).

[31] J. C. Allred, R. N. Lyman, T. W. Kornack and M. V. Romalis, Phys. Rev. Lett. 89, 130801 (2002).

[32] I. K. Kominis, J. C. Allred, T. W. Kornack and M. V. Romalis, Nature 422, 596 (2003).

[33] R. Carminati and J. J. Sáenz, Phys. Rev. Lett. 84, 5156 (2000).

[34] B. Kubala and J. König, Phys. Rev. B 67, 205303 (2003).

[35] P. Facchi and S. Pascazio, J. Phys. A: Math. Theor. 41, 493001 (2008).

[36] K. Koshino and A. Shimizu, Phys. Rep. 412, 191 (2005).

[37] A. G. Kofman and G. Kurizki, Nature 405, 546 (2000).

[38] R. Haberkorn, Molec. Phys. 32, 1491 (1976).

[39] H. M. Wiseman, Quantum Semiclass. Opt. 8, 205 (1996).

[40] J. Jortner, J. Am. Chem. Soc. 102, 6676 (1980).

[41] Y. Kobori et al., Proc. Natl. Acad. Sci. USA 102, 10017 (2005).

[42] V. B. Braginsky and F. Y. Khalili, Quantum Measurement, Cambridge University Press, Cambridge, 1995.

[43] W. M. Itano, Phys. Rev. A 41, 2295 (1990).

[44] S. A. Gurvitz, Phys. Rev. B 56, 15215 (1997).

[45] K. Jacobs and D. A. Steck, Contemp. Phys. 47, 279 (2006).

[46] D. Anglos, Photoinduced Intrapeptide Electron Transfer Involving Novel Donor and Acceptor Amino Acids: A Triplet State Approach, Ph.D. Thesis, Cornell University, 1994. 